\title{
Fear of the Dark: Diazo Printing by Photochemical Decomposition of Aryldiazonium Tetrafluoroborates
}

Fabio Parmeggiani*

5 Department of Chemistry, Materials and Chemical Engineering "G. Natta", Politecnico di Milano, Piazza Leonardo Da Vinci 32, I-20131, Milano, Italy

\section{ABSTRACT}

The photochemical decomposition of aryldiazonium salts is the basis of the "diazo printing" technique, which has been the only means to reproduce technical drawings for many years. Several demonstrations of the procedure have been developed, but they all rely either on commercially available photosensitive paper, which is almost impossible to obtain now, or on the preparation of diazonium salts from very toxic and carcinogenic aromatic amines. A variation of the procedure is reported, taking advantage of a recent synthetic method based on a one-pot deacylation-diazotization of acetanilides, which are non-carcinogenic, much less toxic than the corresponding amines, and insensitive to air oxidation. In the demonstration, $p$-ethoxybenzenediazonium tetrafluoroborate, easily synthesized from phenacetin, is combined with a coupling component, yielding photosensitive paper that can be printed by exposure to UV light through a suitable template (flat objects, drawings, etc.), and developed with ammonia. With the three couplers suggested it is possible to obtain red, dark yellow and purple prints.

\section{TABLE OF CONTENTS/GRAPHIC ABSTRACT}

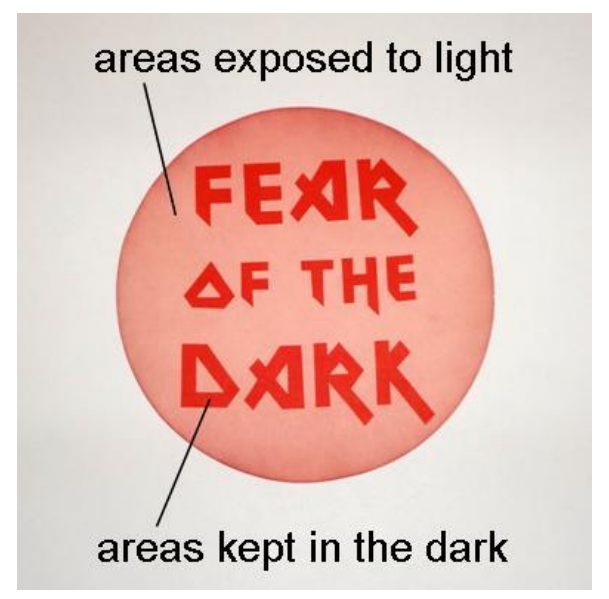

\section{KEYWORDS}

General Public - Upper-Division Undergraduates - Demonstrations - Organic Chemistry Hands-on learning/Manipulatives - Amides - Amines/Ammonium Compounds 


\section{INTRODUCTION}

Aryldiazonium salts are extremely versatile intermediates in the organic synthesis of aromatic compounds. ${ }^{1}$ They are easily formed from the corresponding amines and may undergo a variety of reactions, such as the replacement of the diazonium group with hydrogen, halides, cyanide or hydroxyl groups, with the loss of molecular nitrogen (eq. 1 ), and the coupling with electron-rich aromatic rings to form azoic dyes (diazocoupling, eq. 2). Many of these reactions have been adopted as laboratory experiments or classroom demonstrations, due to their considerable educational value. ${ }^{2}$

$$
\begin{aligned}
& \mathrm{ArN}_{2}^{+}+\mathrm{MX} \rightarrow \mathrm{ArX}+\mathrm{M}^{+}+\mathrm{N}_{2} \\
& \mathrm{ArN}_{2}{ }^{+}+\mathrm{Ar} \mathrm{H}(\text { coupler }) \rightarrow \mathrm{Ar}-\mathrm{N}=\mathrm{N}-\mathrm{Ar}^{\prime}+\mathrm{H}^{+}
\end{aligned}
$$

One of the properties of diazonium salts that is often neglected in introductory organic chemistry courses is their sensitivity to light. These compounds can decompose photochemically, due to the action of light of a suitable wavelength (usually 350-450 $\mathrm{nm}$ ), yielding nitrogen and the corresponding aryl cation (eq. 3), which almost immediately reacts with water or other nucleophiles forming a phenol (eq. 4) or other subproducts.

$$
\begin{aligned}
& \mathrm{ArN}_{2}^{+}+\mathrm{hv} \rightarrow \mathrm{Ar}^{+}+\mathrm{N}_{2} \\
& \mathrm{Ar}^{+}+\mathrm{H}_{2} \mathrm{O} \rightarrow \mathrm{ArOH}+\mathrm{H}^{+}+\text {subproducts }
\end{aligned}
$$

The latter photodegradation mechanism lies at the heart of the printing technique known as "diazo printing" (or "whiteprint", as opposed to the older "blueprint" process), patented in the 1890s and well-known for the reproduction of technical drawings for decades. $^{3}$ In this technique, a sheet of paper or film is coated with a mixture of a diazonium salt and a coupling component in the presence of an acid that prevents the coupling reaction. Then, the paper is exposed to UV light through the drawing that has to be reproduced: where the light shines on the paper, the diazonium salt is decomposed, while no reaction occurs in dark areas. After exposure, the paper is fed to a developing chamber saturated with ammonia vapor that neutralizes the acid and allows the diazocoupling reaction to occur between the remaining diazonium salt and the coupling agent, forming a brightly colored diazo dye.

Several brilliant demonstrations of this process have been reported in the literature, but they rely on commercial photosensitive paper or film (which is harder and harder to find).$^{4}$ On the other hand, the remaining few demonstrations or laboratory experiments that allow an instructor or students to prepare their own photosensitive paper all employ diazonium tetrafluoroborates that must be synthesized from carcinogenic and highly toxic aromatic amines (e.g., $p$-anisidine or $p$-dimethylaminoaniline). ${ }^{5}$ Even though the reaction is very effective and of undoubted educational value, the latter chemicals are definitely hazardous and worth substituting with less dangerous alternatives.

75 A variation of this valuable and practical demonstration is described, preserving the impressive visual effect but employing a harmless and inexpensive starting material.

\section{DEVELOPMENT OF THE DEMONSTRATION}

Recently, a new preparative protocol for the synthesis of aryldiazonium tetrafluoroborates was developed for application in Heck reactions. ${ }^{6}$ The procedure is based on the one-pot deacylation-diazotization of many substituted acetamides such as paracetamol 1, p-methoxyacetanilide 2 and phenacetin 3 (Scheme 1). Besides being 
non-carcinogenic and much less toxic than the corresponding amines, acetanilides show several advantages: they are usually crystalline, easily purified by crystallization, and insensitive to air oxidation. At the end of the one-pot two-step procedure, the diazonium tetrafluoroborate (4-6) spontaneously precipitates and is collected by vacuum filtration. It can be purified by recrystallization from boiling methanol, and stored almost indefinitely in a dark and cool place.

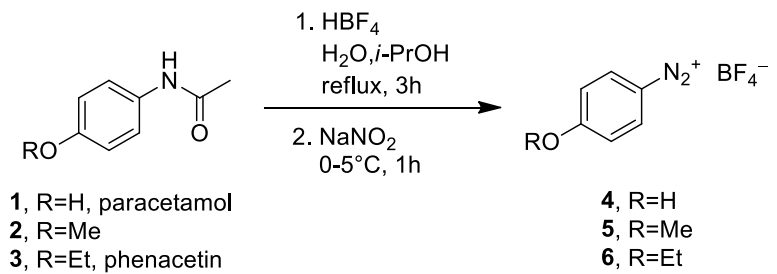

Scheme 1. Synthesis of aryldiazonium tetrafluoroborates 4-6 from acetanilides 1-3, according to the recent deacylationdiazotization protocol.

The diazo printing procedure does not work properly with $p$ hydroxybenzenediazonium salt $\mathbf{4}$, likely due to its higher reactivity towards oxidation and other side-reactions. On the other hand, the $p$-methoxy analogue $\mathbf{5}$ is very effective, but in this case the precursor $\mathbf{2}$ is not readily available and has to be synthesized from 1, employing highly toxic and carcinogenic reagents such as $\mathrm{Me}_{2} \mathrm{SO}_{4}$ or MeI. In constrast, the p-ethoxy analogue $\mathbf{6}$ is equally effective, and the precursor $\mathbf{3}$ is commercially available and inexpensive, due to its widespread use as an analgesic and antipyretic.

Of several couplers screened in order to obtain different colors and avoiding those that pose a serious health risk (such as a-naphthol, $N, N$-dimethylaniline and naphthylamines), the best results were obtained with $\beta$-naphthol $\mathbf{7}$, resorcinol $\mathbf{8}$, and 4amino-5-hydroxynaphthalene-2,7-disulfonic acid $\mathbf{9}$ (also known as "H-acid" in the dye 105 industry). These combine with 6 to yield red, dark yellow and purple diazo dyes 10-12, respectively (Figure 1). 


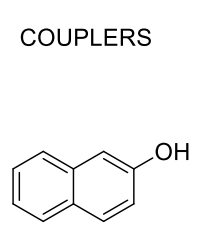

7, $\beta$-naphthol

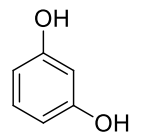

8, resorcinol

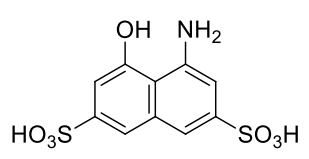

9, "H-acid"
DIAZO DYES

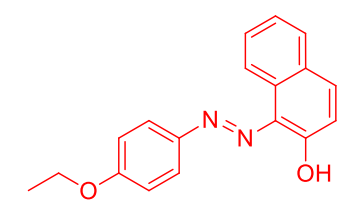

10

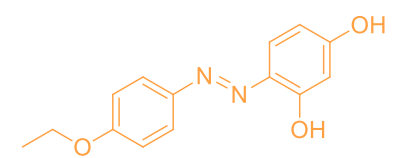

11

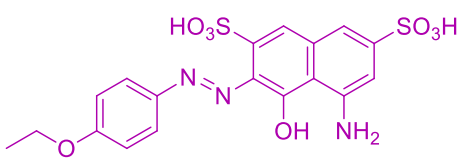

12

Figure 1. Structures of the couplers employed and of the corresponding diazo dyes formed.

The procedure for the preparation of photosensitive paper is very simple. The diazonium salt $\mathbf{6}$ and a coupler 7-9 are dissolved in methanol (acidified with sulfuric acid to suppress the coupling), filter paper is soaked with the solution and left to dry in the dark.

To demonstrate the photochemical decomposition, one sample of paper is kept in the dark and another is exposed to long wavelength UV light or bright sunlight. The color of the irradiated paper changes only moderately. When the two samples are placed in a developing chamber saturated with vapors of concentrated ammonia solution, the non-exposed paper turns brightly colored, while the other does not change color appreciably (Scheme 2). Diazo printing is demonstrated by placing small opaque objects on the paper before exposing it to light (Figure 2 illustrates the stages of the procedure). Figure 3 shows typical results that are obtained with the three couplers and different templates. Many things can be used as a template, but flat objects are best because the image will be unclear if the template does not touch the paper completely (see Figure S1 in the Supporting Information). It is also very easy to make a message appear on the paper using stencils or letters cut out from paper or cardboard (see for instance the Graphic Abstract or Figures S2 and S3 in the Supporting Information). Another option is to write the message with a black marker on a glass plate to be used as a template; this is the most faithful reproduction of the original diazo printing process. 


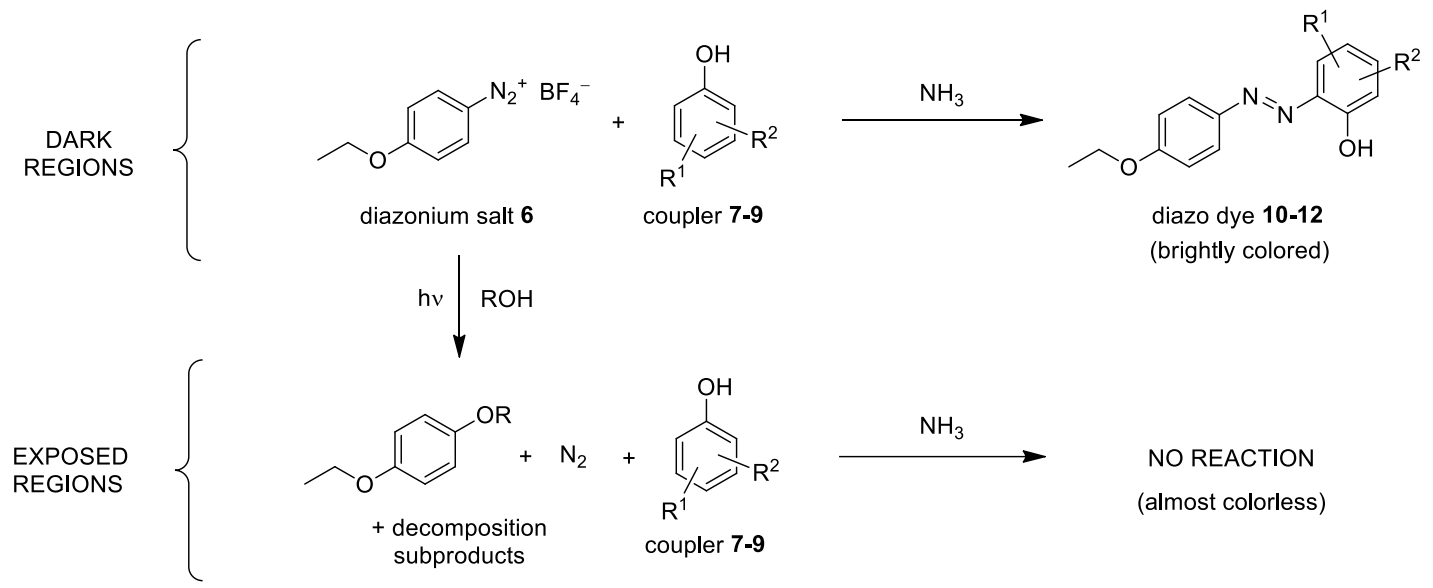

Scheme 2. Photochemical decomposition of $p$-ethoxybenzenediazonium tetrafluoroborate 6 and exploitation of the reaction in diazo printing.

140
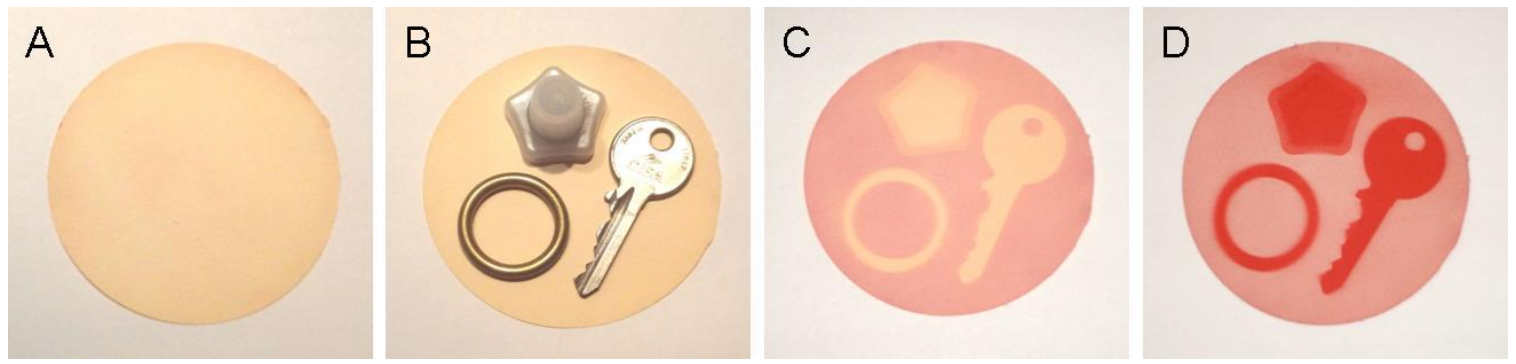

Figure 2. Demonstration of diazo printing: (A) dry paper treated with the photosensitive solution of 6 and 7 ; (B) objects placed on the paper; (C) after UV irradiation, (D) after development with ammonia. 


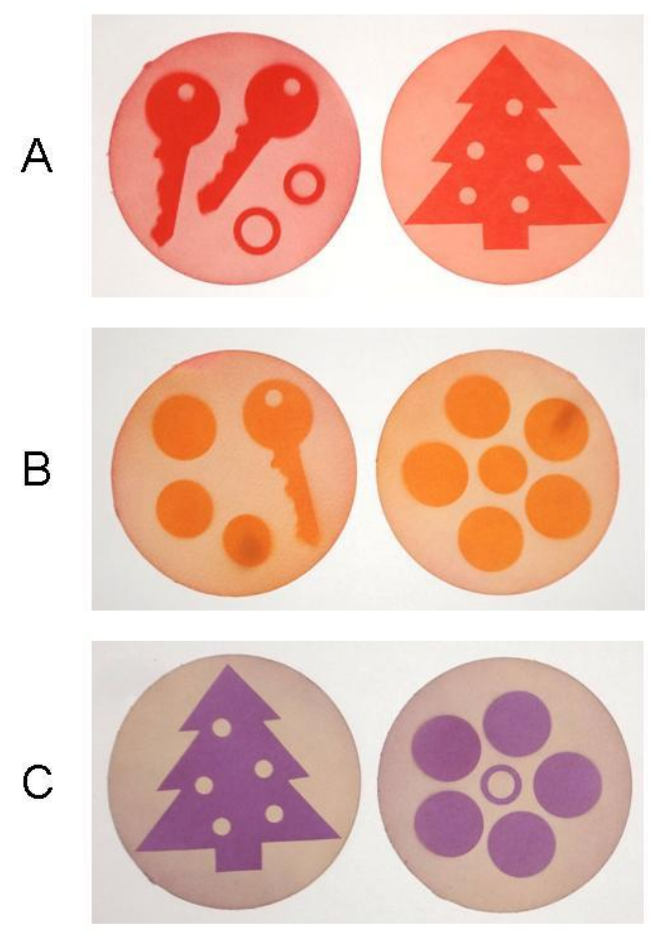

Figure 3. Examples of developed prints obtained with keys, coins, washers and a Christmas tree stencil: (A) 6+7; (B) 6+8; (C) $6+9$.

\section{EXPERIMENTAL PROCEDURE}

Synthesis of $p$-ethoxybenzenediazonium tetrafluoroborate 6

Phenacetin $(5.0 \mathrm{~g}, 27.9 \mathrm{mmol})$ is suspended in a solution of conc. $\mathrm{HBF}_{4}(8.0 \mathrm{~mL}$, $48 \% \mathrm{w} / \mathrm{w})$, water $(8.0 \mathrm{~mL})$ and $i-\mathrm{PrOH}(8.0 \mathrm{~mL})$. The suspension is heated under reflux for $3 \mathrm{~h}$ and cooled in an ice bath (partial solidification occurs). Under efficient magnetic stirring, solid $\mathrm{NaNO}_{2}(3.1 \mathrm{~g}, 44.9 \mathrm{mmol})$ is added in portions, keeping the temperature below $5^{\circ} \mathrm{C}$. During the addition, the mixture becomes homogeneous and towards the end the diazonium salt begins to precipitate. The stirring is continued on ice for $30 \mathrm{~min}$, then the brown solid is filtered under vacuum and washed with $\mathrm{Et}_{2} \mathrm{O}(4 \times 25 \mathrm{~mL})$. After drying as much as possible, the solid is recrystallized from boiling $\mathrm{MeOH}(25 \mathrm{~mL})$ in subdued light conditions, filtered again under vacuum and dried carefully. The crystalline product is stable almost indefinitely if it is kept dry and away from sources of light and heat. Yield: $4.1 \mathrm{~g}, 62 \% .{ }^{1} \mathrm{H}$ NMR (400 MHz, DMSO- $\left.d_{6}\right): 8.59$ (m, 2H, arom.), 7.46 (m, 2H, arom.), 4.35 (q, $\left.J=7.0 \mathrm{~Hz}, 2 \mathrm{H}, \mathrm{CH}_{2}\right), 1.40$ (t, $\left.J=7.0 \mathrm{~Hz}, 3 \mathrm{H}, \mathrm{CH}_{3}\right) .{ }^{13} \mathrm{C}$ NMR (100.6 MHz, DMSO- $\left.d_{6}\right): 168.0,136.0,117.4,102.8,65.9,13.9$.

\section{Preparation of photosensitive paper}

The coupler, either $\beta$-naphthol (72 $\mathrm{mg}, 0.5 \mathrm{mmol})$, resorcinol $(55 \mathrm{mg}, 0.5 \mathrm{mmol}$ ), or $\mathrm{H}$-acid $(96 \mathrm{mg}, 0.3 \mathrm{mmol})$, is dissolved in $\mathrm{MeOH}(10 \mathrm{~mL})$ and the solution is acidified with conc. $\mathrm{H}_{2} \mathrm{SO}_{4}$ (2 drops). Avoiding exposure to bright light sources, the diazonium salt $6(118 \mathrm{mg}, 0.5 \mathrm{mmol})$ is dissolved in the solution, and the solution is dripped on several pieces of filter paper until they are soaked completely. The pieces are dried for $30 \mathrm{~min}$ in complete darkness, and stored in sealed, light-proof envelopes. 


\section{Demonstration of the diazo printing}

The piece of paper is shown to have a yellowish color. A few opaque objects, such as coins, keys, rings (provided by the audience, if desired), are placed on the paper and the latter is irradiated with a UV lamp $(366 \mathrm{~nm})$ for $20 \mathrm{~min}$ or in bright sunlight for $2 \mathrm{~h}$. The objects are removed and the paper can be briefly passed around the audience to show that only a faint image is visible. Then, the paper is placed in a transparent container (e.g., a TLC developing chamber or a large Petri dish) containing a few $\mathrm{mL}$ of conc. ammonia solution in a smaller container (e.g., a beaker or a watchglass), preventing direct contact between the paper and the solution. Within $5 \mathrm{~min}$, the non-exposed area of the paper will become colored, while the background will develop only a weak brownish color (see the Supporting Information for a videoclip). After the development, the piece of paper can be passed around in a transparent envelope.

\section{HAZARDS}

Diazonium salts with poorly coordinating anions (e.g., $\mathrm{BF}_{4}^{-}$and $\mathrm{PF}_{6}{ }^{-}$) are relatively stable and can be stored for long periods of time if pure and dry. However, they should still be treated as potentially explosive materials. Concentrated tetrafluoroboric acid and all the organic compounds employed should be handled with suitable gloves. Adequate ventilation must be provided when working with concentrated ammonia solution.

\section{SUMMARY}

The procedure described is meant to be used as a demonstration of the photochemical decomposition of diazonium tetrafluoroborates for introductory organic chemistry courses. The instructor can prepare the photosensitive paper in advance, perform the exposure step while lecturing on diazonium chemistry and develop the image at the very end. However, since the procedure is particularly easy and does not call for carcinogenic reagents or dedicated glassware, it can also be adapted as a laboratory experiment for students (specific directions are given in the Supporting Information).

\section{ASSOCIATED CONTENT}

Supporting Information

Videoclip of the development step, additional figures, ${ }^{1} \mathrm{H}$ and ${ }^{13} \mathrm{C}$ NMR spectra of $\mathbf{6}$, instructor's notes, students handout for use as a laboratory experiment. This material is available via the Internet at http://pubs.acs.org.

\section{AUTHOR INFORMATION}

Corresponding Author

210 *E-mail: fabio.parmeggiani@polimi.it

Notes

The author declares no competing financial interest.

\section{ACKNOWLEDGMENT}

The author is grateful to Elisabetta Brenna and Francesco G. Gatti for valuable discussion.

\section{REFERENCES}

1. (a) Zollinger, H. Diazo Chemistry, Vol. I: Aromatic and Heteroaromatic Compounds; VCH: Weinheim, Germany, 1994; (b) Wulfman, D. S. The Chemistry of Diazonium and Diazo Compounds; Wiley: New York, USA, 1978. 
2. (a) Zollinger H. Lecture Demonstration of a Kinetic Isotope Effect. J. Chem. Educ. 1957, 34, 249; (b) Stone, D. B. Diazonium Salts as Spot Test and Synthetic Reagents. J. Chem. Educ. 1971, 48, 413-414; (c) Smith, N. H. P. A Rapid and Convenient Lecture Demonstration of Dyeing with Azo Colors. J. Chem. Educ. 1973, 50, 790-791; (d) Zangel, M.; McKee, J. R. The Synthesis of Methyl Salicylate: Amine Diazotization. J. Chem. Educ. 1988, 65, 1106; (e) Costas-Costas, U.; Pazo-Llorrente, R.; González-Romero, E.; Bravo-Díaz, C. Dediazoniations in Water: an Integrated Physical Organic Chemistry Experiment. J. Chem. Educ. 2000, 77, 384-386; (f) Gung, B. W.; Taylor, R. T. Parallel Combinatorial Synthesis of Azo Dyes: A Combinatorial Experiment Suitable for Undergraduate Laboratories. J. Chem. Educ. 2004, 81, 1630-1632; (g) Gregor, R. W.; Goj, L. A. Solvent-Free Synthesis of 2,2'-Dinitrobiphenyl: An Ullmann Coupling in the Introductory Organic Laboratory. J. Chem. Educ. 2011, 88, 331333; (h) Van Rens, L.; Van Dijk, H.; Mulder, J.; Nieuwland, P. Using a Web Application to Conduct and Investigate Syntheses of Methyl Orange Remotely. J. Chem. Educ. 2013, 90, 574-577

3. (a) Verry, H. R. Document Copying and Reproduction Processes; Fountain Press: London, 1972; (b) Benbrook, C. H. The Diazotype Process. J. Am. Soc. Inf. Sci. Technol. 1957, 8, 8188.

4. (a) Smith, F. E. Lensless Copying with Sensitized Papers. J. Chem. Educ. 1954, 31, 351-353; (b) Alyea, H. N. Dem. 359 - Diazo-printing. J. Chem. Educ. 1967, 44, A145.

5. (a) Osterby, B. The Diazo Copying Process. J. Chem. Educ. 1989, 66, 1026-1028; (b) Fanghänel, E. Photochemical Decomposition of Aromatic Diazonium Salts. Pure \& Appl. Chem. 1992, 64 (9), 1362.

6. (a) Schmidt, B.; Berger, R.; Hölter, F. Functionalized Alkoxy Arene Diazonium Salts from Paracetamol. Org. Biomol. Chem. 2010, 8, 1406-1414; (b) Schmidt, B.; Hölter, F.; Berger, R.; Jessel, S. Mizoroki-Heck Reactions with 4-Phenoldiazonium Salts. Adv. Synth. Catal. 2010, 352, 2463-2473. 